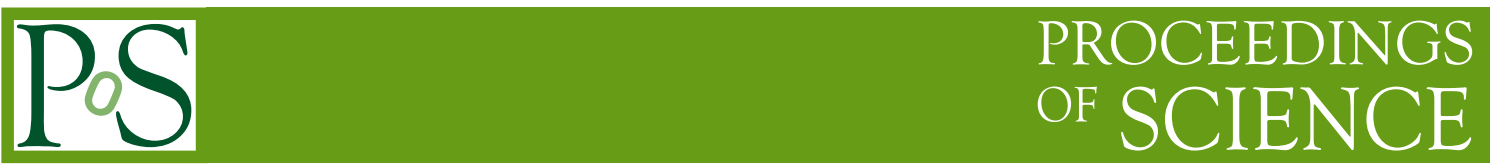

\title{
Coulomb corrections to DIS off heavy nuclei
}

\author{
Kirill Tuchin*† \\ Department of Physics and Astronomy, Iowa State University, Ames, Iowa, 50011, USA \\ E-mail: tuchin@iastate.edu
}

\begin{abstract}
An essential part of experimental program at the future Electron Ion Collider is the study of the nuclear structure and dynamics at low $x$. DIS at low $x$ is characterized by large longitudinal coherence length that by far exceeds radii of heavy nuclei. The coherent behavior is essential feature of the nuclear matter at low $x$. This pertains not only to the strong interactions, but also to electromagnetic ones. Coherent interactions of a projectile with nucleons in a heavy nucleus are characterized by parameters $\alpha_{s}^{2} A^{1 / 3} \sim 1$ and $\alpha Z \sim 1$ in strong and electromagnetic interactions respectively. Contributions exhibiting non-trivial dependence on $\alpha Z$ are called the Coulomb corrections. We compute the Coulomb corrections to the cross sections of the semi-inclusive and diffractive DIS. We show that they violate the geometric scaling in a wide range of photon virtualities and is weakly $x$-independent. In heavy nuclei at low $Q^{2}$ the Coulomb correction to the total and diffractive cross sections is about $20 \%$ and $40 \%$ correspondingly.
\end{abstract}

XXIII International Workshop on Deep-Inelastic Scattering,

27 April - May 12015

Dallas, Texas

\footnotetext{
* Speaker.

${ }^{\dagger}$ This work was supported in part by the U.S. Department of Energy under Grant No. DE-FG02-87ER40371.
} 


\section{Why electromagnetic contribution is important}

DIS at low $x$ is characterized by large longitudinal coherence length that by far exceeds radii of heavy nuclei. In QCD, color fields of nucleons in a heavy nucleus fuse to create an intense coherent color field which has fundamental theoretical and phenomenological importance. Since nuclear force is short-range, only nucleons along the same impact parameter add up to form the coherent field. Because the QCD contribution to the scattering amplitude at high energy is approximately imaginary, it is proportional to $\alpha_{s}^{2}$. Thus, the parameter that characterizes the color-coherent field is $\alpha_{s}^{2} A^{1 / 3} \sim 1$, where $A$ is atomic weight.

Along with strong color field, heavy-ions also posses strong electromagnetic Coulomb field. The electromagnetic force is long range, therefore all $Z$ protons of an ion contribute to the field. The QED contribution to the scattering amplitude is approximately real. As a result, the parameter that characterizes the coherent electromagnetic field is $\alpha Z \sim 1$. Since both parameters $\alpha_{s}^{2} A^{1 / 3}$ and $\alpha Z$ are of the same order of magnitude in heavy ions, electromagnetic force must be taken into account along with the color one. This observation is a direct consequence of coherence which enhances the electromagnetic contribution by a large factor $Z$. Such electromagnetic contributions are called the Coulomb corrections.

In this article I focus on the Coulomb corrections to the total semi-inclusive and diffractive cross sections. At low $x$ the total $\gamma^{*} A$ cross section can be expressed in terms of the total dipolenucleus cross section $\hat{\sigma}$ as follows (see e.g. [1])

$$
\sigma_{T / L}\left(x, Q^{2}\right)=\frac{1}{4 \pi} \int_{0}^{1} d z \int d^{2} r \Phi_{T / L}(r, z) \hat{\sigma}(x, r),
$$

where $Q^{2}$ is the photon virtuality. The light-cone wave functions for transverse and longitudinal polarizations of photon are given by

$$
\begin{array}{r}
\Phi_{T}=\sum_{f} \frac{2 \alpha N_{c}}{\pi}\left\{\left[z^{2}+(1-z)^{2}\right] a^{2} K_{1}^{2}(a r)+m_{f}^{2} K_{0}^{2}(a r)\right\}, \\
\Phi_{L}=\sum_{f} \frac{2 \alpha N_{c}}{\pi} 4 Q^{2} z^{2}(1-z)^{2} K_{0}^{2}(a r),
\end{array}
$$

where $m_{f}$ is quark mass, $z$ is the fraction of the photon's light-cone momentum carried by the quark, $r$ is the size of the $q \bar{q}$ dipole in the transverse plane and $a^{2}=z(1-z) Q^{2}+m_{f}^{2}$. ${ }^{1}$ The dipole cross section $\hat{\sigma}$ depends non-trivially on $A$ and $Z$. In the following sections I explain how it can be calculated. More details can be found in $[2,3]$.

\section{Nuclear dependence of the dipole cross section $\hat{\sigma}$.}

At high energies, interaction of the projectile proton with different nucleons is independent inasmuch as the nucleons do not overlap in the longitudinal direction. This assumption is tantamount to taking into account only two-body interactions, while neglecting the many-body ones [5].

\footnotetext{
${ }^{1}$ The relationship between the cross section $\sigma=\sigma_{T}+\sigma_{L}$ and $F_{1}, F_{2}$ structure functions is non-trivial due to large Coulomb corrections to the leptonic tensor [4].
} 
In this approximation we can write the dipole-nucleus elastic scattering amplitude as

$$
\Gamma(\boldsymbol{b})=1-\exp \left\{-\sum_{a}\left\langle\Gamma^{(1)}(\boldsymbol{b})\right\rangle\right\}
$$

where $\Gamma^{(1)}(\boldsymbol{b})$ is the dipole-nucleon elastic scattering amplitude and $\langle\ldots\rangle$ denotes average over the nucleon position in the nucleus. For the sake of brevity, dependence of the scattering amplitudes on $x$ and $r$ is not explicitly indicated. The total dipole cross section can be computed using the optical theorem as follows

$$
\hat{\sigma}=2 \int d^{2} b \operatorname{Im}[i \Gamma(\boldsymbol{b})] .
$$

Strong and electromagnetic contributions decouple in the elastic scattering amplitude at the leading order in respective couplings:

$$
\Gamma^{(1)}=\Gamma_{s}^{(1)}+\Gamma_{e m}^{(1)} .
$$

This is because $i \Gamma_{e m}^{(1)}$ is real, while $i \Gamma_{s}^{(1)}$ is imaginary, as discuss below. Owing to (2.3) we can cast (2.1) in the form

$$
\Gamma(\boldsymbol{b})=1-\exp \left\{-A\left\langle\Gamma_{s}^{(1)}(\boldsymbol{b})\right\rangle-Z\left\langle\Gamma_{\text {em }}^{(1)}(\boldsymbol{b})\right\rangle\right\} .
$$

Averaging over the nucleus wave function is done as follows

$$
\left\langle\Gamma_{s}^{(1)}(\boldsymbol{b})\right\rangle=\frac{1}{A} \int_{-\infty}^{\infty} d z_{a} \int d^{2} b_{a} \rho\left(\boldsymbol{b}_{a}, z_{a}\right) \Gamma_{s}^{(1)}\left(\boldsymbol{b}-\boldsymbol{b}_{a}\right),
$$

where $\rho$ is the nuclear density. Neglecting the diffusion region, nuclear density is approximately constant $\rho=A /\left(\frac{4}{3} \pi R_{A}^{3}\right)$ for points inside the nucleus and zero otherwise. The range of the nuclear force is about a fm, which is much smaller than the radius $R_{A}$ of a heavy nucleus. Therefore, $\boldsymbol{b} \approx \boldsymbol{b}_{a}$ and

$$
\left\langle\Gamma_{s}^{(1)}(\boldsymbol{b})\right\rangle=\frac{1}{A} 2 \sqrt{R_{A}^{2}-b^{2}} \pi R_{A}^{2} \rho \Gamma_{s}^{(1)}(0)=\frac{2 C_{F}}{A N_{c}} \rho T(b) \frac{1}{2} \pi r^{2} \alpha_{s}^{2} \ln \frac{1}{r \mu},
$$

where $T(b)=2 \sqrt{R_{A}^{2}-b^{2}}$ is the thickness function and $\mu$ is an infra-red scale. It follows from (2.6) that $A\left\langle\Gamma_{s}^{(1)}\right\rangle \sim \alpha_{s}^{2} A^{1 / 3}$, which implies that (2.4) sums up terms of order $\alpha_{s}^{2} A^{1 / 3} \sim 1$ at $\alpha_{s} \ll 1$. Indeed, the leading strong-interaction contribution to the $\gamma^{*} N$ elastic scattering amplitude corresponds to double-gluon exchange. Note also, that the corresponding $\left\langle i \Gamma_{s}^{(1)}\right\rangle$ is purely imaginary.

To calculate the electromagnetic contribution to the $\gamma^{*} N$ scattering, note that the proton density in the nucleus is $Z \rho / A$. Hence

$$
\begin{array}{r}
\left\langle\Gamma_{\text {em }}^{(1)}(\boldsymbol{b})\right\rangle= \\
=\frac{1}{Z} \int_{-\infty}^{\infty} d z_{a} \int d^{2} b_{a} \frac{Z}{A} \rho\left(\boldsymbol{b}_{a}, z_{a}\right) \Gamma_{\text {em }}^{(1)}\left(\boldsymbol{b}-\boldsymbol{b}_{a}\right) \\
=\frac{1}{i A} \rho 2 \alpha \int d^{2} b_{a} T\left(b_{a}\right) \ln \frac{\left|\boldsymbol{b}-\boldsymbol{b}_{\boldsymbol{a}}-\boldsymbol{r} / 2\right|}{\left|\boldsymbol{b}-\boldsymbol{b}_{a}+\boldsymbol{r} / 2\right|} .
\end{array}
$$

The leading electromagnetic contribution to elastic $\gamma^{*} N$ scattering amplitude arises from one photon exchange; the corresponding $\left\langle i \Gamma_{e m}^{(1)}\right\rangle$ is purely real. We note, that (2.5) sums up terms of order $\alpha Z \sim 1$ at $\alpha \ll 1$. Had we been interested in purely electromagnetic scattering (e.g. of $e^{-} e^{+}$instead of $q \bar{q}$ ) we could have approximated $b \gg b_{a} \sim R_{A}$ owing to the long-range nature of the Coulomb potential. That would have yielded the well-known Bethe-Heitler-Maximon result $[6,7]$ as shown 
in [8]. However, in DIS $b \sim R_{A}$ and no such approximation is possible. It follows from (2.2) and (2.4) that

$$
\hat{\sigma}=2 \int d^{2} b\left\{1-\exp \left[-A\left\langle\Gamma_{s}^{(1)}(\boldsymbol{b})\right\rangle\right] \cos \left[Z\left\langle i \Gamma_{\text {em }}^{(1)}(\boldsymbol{b})\right\rangle\right]\right\} .
$$

Integrals in (2.8) and (2.9) can be analytically calculated in a simple but quite accurate "cylindrical nucleus" model (see e.g. $[9,10]$ ), which approximates the nuclear thickness function by the step function, viz. $T(b)=2 R_{A}$ if $b<R_{A}$ and zero otherwise. The result is [2]

$$
\begin{gathered}
\hat{\sigma}(x, r)=\hat{\sigma}_{s}(x, r)+\hat{\sigma}_{e m}(x, r), \\
\hat{\sigma}_{s}(x, r)=2 \pi R_{A}^{2}\left\{1-\exp \left[-\frac{1}{4} \tilde{Q}_{s}^{2}(x) r^{2}\right]\right\}, \\
\hat{\sigma}_{e m}(x, r)=4 \pi r^{2}(\alpha Z)^{2} \ln \frac{W^{2}}{4 m_{f}^{2} m_{N} R_{A}},
\end{gathered}
$$

where $m_{N}$ is nucleon mass, $W$ is the $\gamma^{*} A$ center-of-mass energy given by $W^{2}=Q^{2} / x+m_{N}^{2}$ and $\tilde{Q}_{s}^{2}$ is the quark saturation momentum (see e.g. [1]).

Logarithm that appears in (2.12) is the result of integration over the impact parameter from $R_{A}$ up to a cutoff $b_{\max }$, which delimits the region of validity of the Weizsäcker-Williams approximation. It is given by $b_{\max }=\max \left\{W^{2} z(1-z) /\left(m_{N}\left(m_{f}^{2}+\boldsymbol{k}^{2}\right)\right)\right\}$, where $\boldsymbol{k}$ is the quark's transverse momentum [8]. The largest size of the $q \bar{q}$ dipole, corresponding to the smallest $k$, is $\sim 1 / m_{f}$ due to the confinement. For that reason $b_{\max }$, and hence (2.12), depends on the constituent quark mass $m_{f}$ rather than on the much smaller current quark mass $m_{q}$.

\section{Evolution effects}

Eqs. (2.10)-(2.12) are derived in the quasi-classical approximation where the quark saturation momentum $\tilde{Q}_{s}^{2}$, and hence the QCD contribution to the total cross section, is $x$-independent. At lower $x$, such that $\alpha_{s} \ln (1 / x) \sim 1$, the QCD quantum evolution effects become important and are described by the BK equation $[11,12]$. According to the BK equation the saturation momentum acquires $x$-dependence in the form $\tilde{Q}_{s}^{2} \sim A^{1 / 3} x^{-\lambda}$, where $\lambda$ is a certain positive number [13]. The functional form of the dipole cross section is also evolving with $x$; (2.11) in that case is the initial condition. There are several phenomenological models that describe the evolved dipole cross section. In this article I use the Golec-Biernat-Wusthoff model [14] which retains the functional form of (2.11) while models the saturation momentum as follows

$$
\tilde{Q}_{s}^{2}=Q_{0}^{2}\left(\frac{x_{0}}{x}\right)^{\lambda},
$$

where $Q_{0}=1 \mathrm{GeV}, x_{0}=3.04 \cdot 10^{-4}, \lambda=0.288, R_{A}=R_{p} A^{1 / 3}$ with $R_{p}=3.1 \mathrm{GeV}^{-1}, m_{f}=140 \mathrm{MeV}$, and $N_{f}=3$. If we neglect the electromagnetic term (2.12) and use (2.11) in (1.1), then we immediately observe that the total $\gamma^{*} A$ cross section exhibits the geometric scaling at $Q^{2} \gg m_{f}^{2}$. This is because $x$-dependence arises only through the combination $r^{2} Q_{s}^{2}(x)$, and the dipole size $r$ is determined by $1 / Q$.

That the Coulomb correction violates the geometric scaling is evident from (2.12) which, being an electromagnetic contribution, does not depend on the strength of the color field determined by $\tilde{Q}_{s}^{2}$. Unlike the QCD term (2.11), the QED one (2.12) does not evolve much with $x$. Indeed, at the leading-log oder $\Gamma_{s / e m} \sim(1 / x)^{1+\Delta_{s / e m}}$, where $\Delta_{s}=4 \ln 2\left(\alpha_{s} N_{c} / \pi\right)[15,16]$ and $\Delta_{e m}=(11 / 32) \pi \alpha^{2}$ $[17,18]$. Because $\Delta_{e m} \ll \Delta_{s}$ we can neglect the effect of the QED evolution. 


\section{Total cross section}

Substituting (2.12) into (1.1) and integrating over $r$ we obtain the following analytic expression for the Coulomb correction to the total $\gamma^{*} A$ cross section [3]

$$
\begin{gathered}
\sigma_{e m, T / L}=(\alpha Z)^{2} \ln \frac{W^{2}}{4 m_{f}^{2} m_{N} R_{A}} \sum_{f} \frac{4 \alpha N_{c}}{3 m_{f}^{2}} g_{T / L}\left(Q / m_{f}\right), \\
g_{T}(\eta)=\left[4\left(\eta^{4}+7 \eta^{2}+8\right) \tanh ^{-1}\left(\frac{\eta \sqrt{\eta^{2}+4}}{\eta^{2}+2}\right)-2 \eta \sqrt{\eta^{2}+4}\left(\eta^{2}+8\right)\right]\left[\eta^{3}\left(\eta^{2}+4\right)^{3 / 2}\right]^{-1} \\
g_{L}(\eta)=4\left[\eta \sqrt{\eta^{2}+4}\left(\eta^{2}+6\right)+4\left(\eta^{2}+3\right) \ln \frac{\eta-\sqrt{\eta^{2}+4}}{\eta+\sqrt{\eta^{2}+4}}\right]\left[\eta^{3}\left(\eta^{2}+4\right)^{3 / 2}\right]^{-1}
\end{gathered}
$$

Coulomb correction to the total semi-inclusive cross section (4.1) does not scale with $\tilde{Q}_{s}^{2} / Q^{2}$ and therefore explicitly violate the geometric scaling.

The numerical results are shown in Figs. 1-2. We can see in Fig. 1 that at low $Q^{2}$ the QED correction for uranium nucleus at $x=10^{-4}$ can be as large as $20 \%$ in the total cross section. It is remarkable that the Coulomb correction is non-negligible even at high $Q^{2}$. One should, however, interpret cautiously the results of our calculation at high $Q^{2}$ since the model that we are using does not properly account for the DGLAP evolution. As expected, the relative size of Coulomb corrections increases with the nuclear weight and weakly depends on $x$.

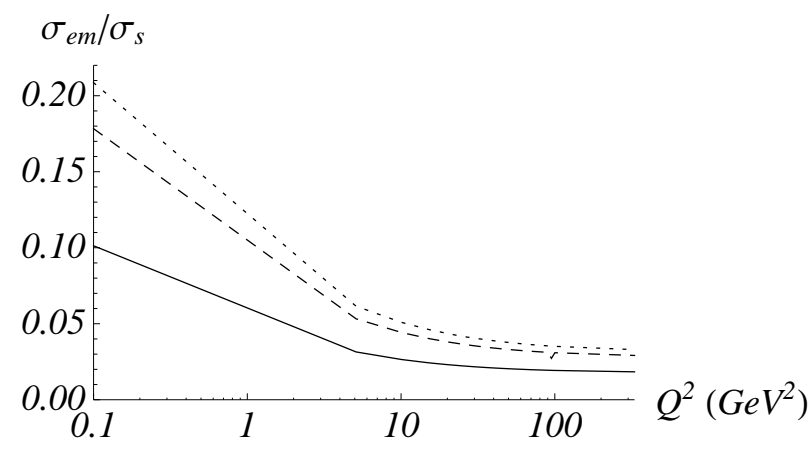

Figure 1: Ratio of QED and QCD contributions to the total $\gamma^{*} A$ cross section at $x=10^{-4}$ as a function of $Q^{2}$ for silver (solid line), gold (dashed line) and uranium (dotted line) nuclei.

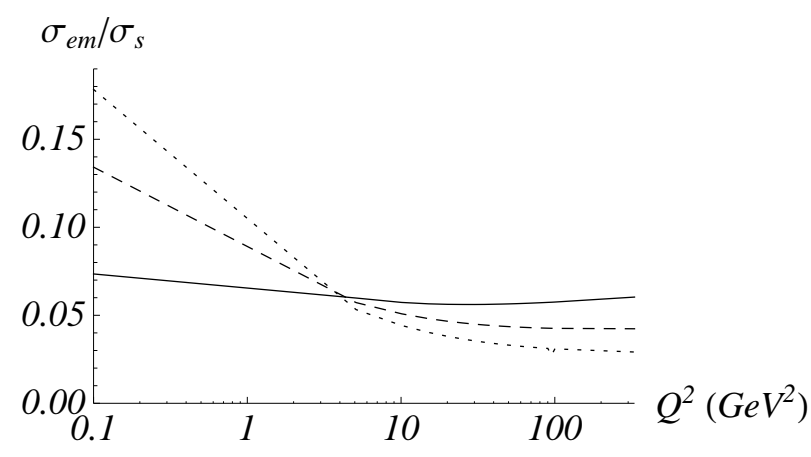

Figure 2: Ratio of QED and QCD contributions to the total $\gamma^{*} A$ cross section as a function of $Q^{2}$ for gold nucleus at $x=10^{-2}$ (solid line), $x=10^{-3}$ (dashed line), $x=10^{-4}$ (dotted line). 


\section{Diffractive cross section}

Total diffractive cross section corresponds to elastic scattering of color dipole on the nucleus. It can be written as

$$
\sigma_{T / L}^{\text {diff }}\left(x, Q^{2}\right)=\frac{1}{4 \pi} \int_{0}^{1} d z \int d^{2} r \Phi_{T / L}(r, z) \hat{\sigma}^{e l}(x, r),
$$

where the total elastic dipole-nucleus cross section reads

$$
\hat{\sigma}^{e l}(x, r)=\int d^{2} b\left|1-\exp \left[-A\left\langle i \Gamma_{s}\right\rangle-Z\left\langle i \Gamma_{e m}\right\rangle\right]\right|^{2}
$$

A simple calculation yields [2]

$$
\hat{\sigma}^{e l}(x, r)=\hat{\sigma}_{s}^{e l}(x, r)+\hat{\sigma}_{e m}(x, r),
$$

where $\hat{\sigma}_{e m}$ is the QED contribution given by (2.12), while the QCD contribution is

$$
\hat{\sigma}_{s}^{e l}(x, r)=\pi R_{A}^{2}\left\{1-\exp \left[-\frac{1}{4} \tilde{Q}_{s}^{2}(x) r^{2}\right]\right\}^{2} .
$$

Numerical results are shown in Figs. 3-4. We can see in Fig. 4 that at low $Q^{2}$ the QED correction for uranium nucleus at $x=10^{-4}$ can be as large as $40 \%$ in the diffractive cross section. The Coulomb corrections to the diffractive cross section are much larger than in semi-inclusive one, see Fig. 3. The reason is that at the leading order in coupling, electromagnetic interaction is elastic.

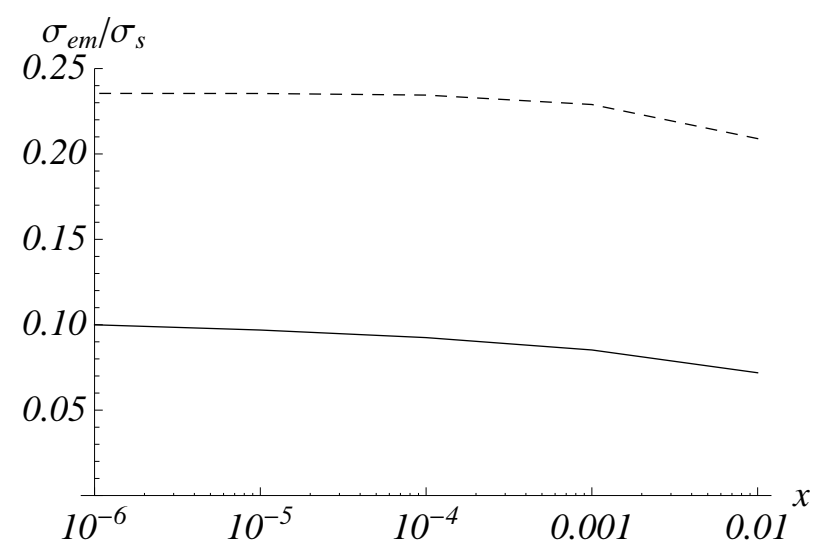

Figure 3: Ratio of QED and QCD contributions to the total (solid line) and diffractive (dashed) $\gamma^{*} A$ cross section as a function of $x$ for gold nucleus at $Q^{2}=1 \mathrm{GeV}^{2}$.

\section{Conclusion: beware of Coulomb corrections}

An essential part of experimental program at the future Electron Ion Collider (EIC) is the study of the nuclear structure and dynamics at low $x$. Nuclear matter at low $x$ exhibits highly coherent behavior, the most striking manifestation of which is the geometric scaling [19, 20, 21, 22, 23]. 


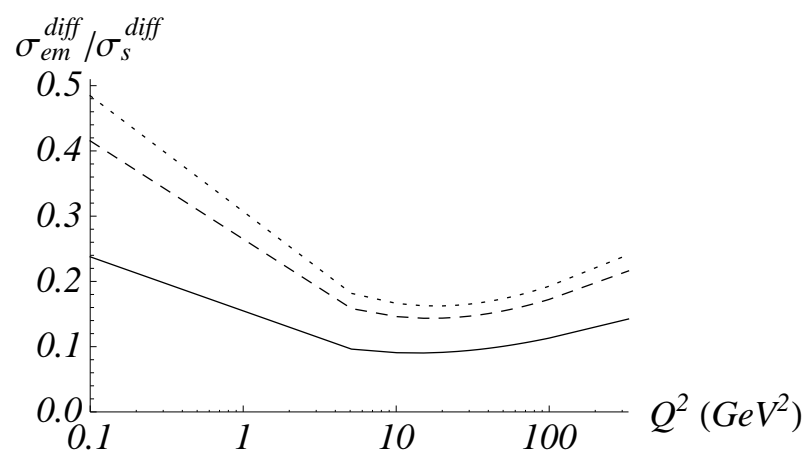

Figure 4: Ratio of QED and QCD contributions to the diffractive $\gamma^{*} A$ cross section at $x=10^{-4}$ as a function of $Q^{2}$ for silver (solid line), gold (dashed line) and uranium (dotted line).

In semi-inclusive DIS the geometric scaling means that the total $\gamma^{*} p$ and $\gamma^{*} A$ cross sections scale with a dimensionless ratio $Q^{2} / \tilde{Q}_{s}^{2}(x)$, where $Q^{2}$ is photon virtuality and $\tilde{Q}_{s}(x)$ is the quark saturation momentum. While the geometric scaling is a signature of coherence in strong interactions, Coulomb corrections is a manifestation of coherence in electromagnetic interactions. Moreover, in DIS off heavy nuclei the two coherence effects are delicately entangled and separate only in a crude cylindrical nucleus model.

Results presented in this work indicate that Coulomb corrections play an important role in the low $x$ DIS off heavy nuclei in a very wide range of $Q^{2}$ and $x$. In particular, they violate the geometric scaling. Since the focus of the EIC program is on the strong interactions, one would like to learn how to subtract the Coulomb corrections from the measured quantities. We showed that Coulomb corrections can in principle be reliably computed. Since we used an oversimplified model for nuclear wave function that allowed us to derive analytical formulas, it is important to refine our estimates by using more realistic nuclear profiles and more sophisticated low $x$ evolution models.

\section{References}

[1] Y. V. Kovchegov and E. Levin, "Quantum chromodynamics at high energy," Cambridge University Press, 2013.

[2] K. Tuchin, Phys. Rev. C 89, no. 2, 024904 (2014) [arXiv:1311.1124 [hep-ph]].

[3] K. Tuchin, Phys. Rev. Lett. 112, no. 7, 072001 (2014) [arXiv:1402.0771 [hep-ph]].

[4] B. Z. Kopeliovich, A. V. Tarasov and O. O. Voskresenskaya, Eur. Phys. J. A 11, 345 (2001)

[5] R. J. Glauber, In *Lo, S.Y. (ed.): Geometrical pictures in hadronic collisions*, 83-182. World Scientific (1987).

[6] H. Bethe and W. Heitler, Proc. Roy. Soc. Lond. A 146, 83 (1934).

[7] H. A. Bethe and L. C. Maximon, Phys. Rev. 93, 768 (1954).

[8] K. Tuchin, Phys. Rev. D 80, 093006 (2009) [arXiv:0907.5189 [hep-ph]].

[9] Y. V. Kovchegov and K. Tuchin, Phys. Rev. D 65, 074026 (2002)

[10] D. Kharzeev, Y. V. Kovchegov and K. Tuchin, Phys. Lett. B 599, 23 (2004) 
[11] I. Balitsky, Nucl. Phys. B 463, 99 (1996)

[12] Y. V. Kovchegov, Phys. Rev. D 60, 034008 (1999)

[13] E. Levin and K. Tuchin, Nucl. Phys. A 693, 787 (2001)

[14] K. J. Golec-Biernat and M. Wusthoff, Phys. Rev. D 59, 014017 (1998)

[15] I. I. Balitsky and L. N. Lipatov, Sov. J. Nucl. Phys. 28 (1978) 822 [Yad. Fiz. 28 (1978) 1597].

[16] E. A. Kuraev, L. N. Lipatov, and V. S. Fadin, Sov. Phys. JETP 45, 199 (1977) [Zh. Eksp. Teor. Fiz. 72 , 377 (1977)].

[17] V. N. Gribov, L. N. Lipatov and G. V. Frolov, Sov. J. Nucl. Phys. 12, 543 (1971) [Yad. Fiz. 12, 994 (1970)].

[18] A. H. Mueller, Nucl. Phys. B 317, 573 (1989).

[19] A. M. Stasto, K. J. Golec-Biernat and J. Kwiecinski, Phys. Rev. Lett. 86, 596 (2001)

[20] L. V. Gribov, E. M. Levin and M. G. Ryskin, Phys. Rept. 100, 1 (1983).

[21] E. Levin and K. Tuchin, Nucl. Phys. B 573, 833 (2000)

[22] E. Levin and K. Tuchin, Nucl. Phys. A 691, 779 (2001)

[23] E. Iancu, K. Itakura and L. McLerran, Nucl. Phys. A 708 (2002) 327 